\title{
Structure of ATP synthase from ESKAPE pathogen Acinetobacter baumannii
}

\author{
Julius K Demmer*,1, Ben P Phillips*,1, O Lisa Uhrig ${ }^{1}$, Alain Filloux², Luke P Allsopp ${ }^{2,3}$, Maike
}

\author{
Bublitz ${ }^{4}$, Thomas Meier ${ }^{\mp, 1,5}$ \\ ${ }^{1}$ Department of Life Sciences \\ Imperial College London \\ Exhibition Road \\ London SW7 2AZ, United Kingdom \\ ${ }^{2}$ MRC Centre for Molecular Bacteriology and Infection \\ Department of Life Sciences \\ Imperial College London \\ London SW7 2AZ, United Kingdom \\ ${ }^{3}$ National Heart and Lung Institute \\ Imperial College, London, UK \\ ${ }^{4}$ Department of Biochemistry \\ University of Oxford \\ South Parks Road \\ Oxford OX1 3QU, United Kingdom \\ ${ }^{5}$ Private University in the Principality of Liechtenstein \\ Triesen, Liechtenstein
}

*These authors contributed equally.

${ }^{\mp}$ corresponding author:

Thomas Meier

t.meier@imperial.ac.uk

One sentence summary: Structure of Acinetobacter baumannii ATP synthase

Keywords: $\mathrm{F}_{1} \mathrm{~F}_{\mathrm{o}}$-ATP synthase, Acinetobacter baumannii, electron cryo-microscopy (cryo-

EM), drug target, ESKAPE, multidrug resistance (MDR), extensively drug resistance (XDR),

bioenergetics

Abbreviations: Adenosine-5'-triphosphate: ATP, electron cryo-microscopy: cryo-EM, multi drug resistance: MDR, extensively drug resistance (XDR) 


\begin{abstract}
Acinetobacter baumannii is a clinically relevant pathogen which causes multi-drug resistant, hospital-acquired infections and is a top priority target for antibiotic development. Cryo-EM structures of the $A$. baumannii $\mathrm{F}_{1} \mathrm{~F}_{\mathrm{o}}-\mathrm{ATP}$ synthase in three conformational states reveal unique features, which represent attractive sites for the development of novel therapeutics.
\end{abstract}

\title{
Article
}

Acinetobacter baumannii is an ESKAPE pathogen ${ }^{1}$ which causes a variety of hospital-acquired infections and severe treatment complications, including SARS-CoV-2 associated pneumonia². A dramatic increase in multi-drug resistance (MDR) in the last decade has placed $A$. baumannii at the top of the WHO priority pathogen list, urgently requiring the development of new drugs with alternative cellular targets ${ }^{3}$. Recent successes in treating MDR bacteria have included novel inhibitors of the $F_{1} F_{0}-A T P$ synthase, with bedaquiline (BDQ) having been approved in 2012 as the only novel last-line antibiotic for MDR Mycobacterium tuberculosis since rifampicin in $1971^{4}$. ATP synthase is highly upregulated in MDR patient strains of $A$. baumannii and, unlike many traditional targets, the enzyme is conditionally essential for survival in biofilms and dormant states ${ }^{5-8}$.

$\mathrm{F}_{1} \mathrm{~F}_{\mathrm{o}}$-ATP synthases are large, membrane-embedded macromolecular complexes that harness energy from the proton-motive force $(p m f)$ and use it to synthesise ATP via a unique rotary mechanism. The simplest forms of ATP synthase are found in bacteria and plant chloroplasts and possess an $F_{1}$ head containing subunits $\alpha_{3} \beta_{3} \gamma \delta \varepsilon$ and an $F_{0}$ motor containing subunits $\mathrm{ab}_{2} \mathrm{c}_{9-15}$. Protons travel through the membrane-embedded a-subunit turning the c-ring. The 
rotating c-ring transfers torque to a central shaft comprising the $\varepsilon$ - and $\gamma$-subunits which protrude into the $\alpha_{3} \beta_{3}$ hexamer, inducing conformational changes in the $F_{1}$ nucleotide binding pockets thus converting the rotary force into ATP synthesis.

In order to identify unique vulnerabilities of the A. baumannii ATP synthase, the complex was purified via affinity-tag and reconstituted into peptidiscs (Figure S1A). ATPase hydrolytic activity assays indicated that the complex was in an 'autoinhibited state' ${ }^{9}$ (Figure S1B) The sample was analysed by single-particle cryo-EM (Figure S1C); 11,490 movies yielded 349,160 particles which were refined into 3 separate states, distinguished by the position of the central stalk and reached overall resolutions of $3.1 \AA$, $4.6 \AA$ and $4.3 \AA$, respectively (Figure S2). Further masked refinements improved the resolution in the $F_{0}$ region of state 1 to $3.7 \AA$ and permitted de novo building of the complex into a composite map. State 1 was then used as a reference to build structures of the remaining two states (Figure S3).

The A. baumannii ATP synthase has a-subunit stoichiometry of $\alpha_{3} \beta_{3} \gamma \delta \varepsilon a b_{2} c_{10}$; its overall architecture resembles that of other bacterial and chloroplast ATP synthases ${ }^{10-12}$ (Figure 1A). The central stalk is rotated by almost exactly $120^{\circ}$ between each of the three conformational states (Figure S4A), as seen previously ${ }^{10}$, suggesting common stable low-energy intermediate states in bacterial ATP synthases.

In $F_{1}$, we observe the canonical 'Walker-Boyer' state geometries in both the 'open' conformation ( $\beta_{\mathrm{E}}$, 'empty') and the 'loose' conformation ( $\beta_{T P}$, with bound Mg-ADP) ${ }^{13,14}$ (Figure 1C). As in Bacillus $\mathrm{PS}^{10}{ }^{10}$, the third $\beta$-subunit ('tight' or $\beta_{\mathrm{DP}}$ ) is in a 'forced open' conformation (denoted as $\beta_{D^{*}}$ ) and we find only weak map features in this region, which we interpret as a 
loosely bound inorganic phosphate ion (Figure 1D). This conformation is induced by an interaction between the $\varepsilon$-subunit and a predominantly hydrophobic loop-helix region in the $\beta_{\text {DP-Subunit }\left({ }^{377} \text { DIIAILGMDE }\right.}{ }^{386}$ ) which overlaps with the highly conserved ${ }^{385}$ DELAEED ${ }^{391}$ motif (Figure S5). The 'forced open' conformation of the $\beta_{D P}$ site is more similar to the open $\beta_{E}$ conformation (all-atom RMSD of $1.5 \AA$ ) than to the loose $\beta_{\text {TP }}$ (all-atom RMSD $\beta_{\text {DP }}$ to $\beta_{\text {TP }} 2.6 \AA$ ) (Figure S5) but is less 'open' than the equivalent site in PS3 (all-atom RMSD A. baumannii $\beta_{\mathrm{DP}}$ * to Bacillus PS3 $\beta_{\mathrm{E}}=0.65 \AA$ ) .

The $A$. baumannii $\varepsilon$-subunit is similar to those seen in other bacteria, containing an extended C-terminal $\alpha$-helix. However, where PS3 $\varepsilon$ terminates, both A. baumannii and Escherichia coli unwind in a short ${ }^{126} \mathrm{AQL}^{128}$ motif (Figure 1B) ${ }^{9,10}$. While the $E$. coli subunit $\varepsilon$ bends here and protrudes 'horizontally' between the $\alpha_{D P}$ and $\beta_{T P}$-subunits, $A$. baumannii $\varepsilon$ continues further upwards into the $F_{1}$ head, forming two more helical turns followed by a 5 -residue extension

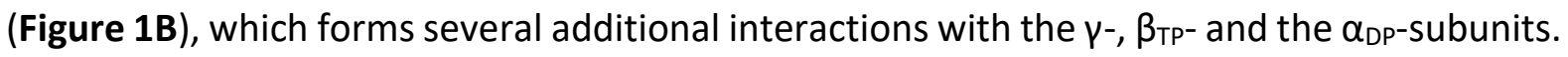
This may further stabilise subunit $\varepsilon$ in the inhibitory 'up' position, which blocks rotation in the hydrolysis direction, while still enabling ATP synthesis ${ }^{10}$ (Figure S4B). This 'ratchet' mechanism is similar to that observed in PS3, but distinct from that in the mycobacterial ATP synthase, which instead relies of the formation of a temporary $\beta$-strand interaction between $\alpha$ and $\varepsilon$ subunits ${ }^{15}$. The stronger blockage may help avoid wasteful ATP hydrolysis, enhancing in vivo host persistence.

The $\gamma \varepsilon$ complex is linked to the $F_{0}$ motor which comprises 10 copies of the c-subunit ( $c_{10}$ ring) and one copy of the proton-conducting a-subunit. Its core fold is highly conserved and resembles other ATP synthase structures, while the proton conducting channels are most 
similar to those in other bacterial a-subunits (Figure 2A). Bacterial ATP synthases contain sequence insertions in the $\mathrm{N}$-terminus of the a-subunit and $A$. baumannii has the largest insertion of the structurally characterised ATP synthases (Figure S6A), resulting in a repositioning of the first short $\alpha$-helix towards the periplasm (Figure 2B). Importantly, this shift relocates the entry site of the periplasmic proton channel: in the mammalian ATP synthase protons enter from behind this mini-helix, whereas in A. baumannii they approach from the front, as viewed from the c-ring (Figure 2B). Positioning of this mini-helix also appears important in determining the channel entry point in other ATP synthases (Figure S7). As several inhibitors of ATP synthases bind to the proton entry channel, including organotin compounds currently employed as pesticides ${ }^{16,17}$, the identification of a distinct entrance may provide an avenue to design specific inhibitors which target bacterial ATP synthases but not those of the mammalian host (Figure 2B, Figure S7).

The $A$. baumannii a-subunit contains an additional loop extension between aH5 and aH6 (Figure 2C and D). The loop extension is formed by predominantly hydrophobic residues plus a charged lysine residue ( ${ }^{200}$ PSNPVAKALLIP ${ }^{211}$ ) and is conserved in the Acinetobacter genus and in the Moraxellaceae family but is fully or partially absent in other ATP synthases (Figure S6A). A structural alignment of a-subunits from several bacterial ATP synthases (Figure 2D) reveals that the $A$. baumannii loop is significantly longer than in other bacteria and may even reach the periplasmic membrane edge (Figure S8). In cells, such proximity may provide privileged access to small molecules and biologics, which is absent in other species. Additionally, K206 appears to stretch towards the bilayer leaflet, suggesting it might even interact with phospholipid headgroups (Figure 2D). 
The success of Bedaquiline (BDQ) in treating tuberculosis has ignited interest in targeting ATP synthases in ESKAPE pathogens. Unlike most ATP synthase inhibitors ${ }^{17}$, BDQ binds with high specificity to mycobacterial ATP synthases. In structures of BDQ bound to the M. smegmatis ATP synthase, the highest affinity binding sites were found at the interface between $c_{10}$ and the a-subunit (Figure $2 \mathrm{C})^{15}$. Furthermore, the broad-spectrum antimalarial antibiotic mefloquine and the new antibiotic lead tomatidine ${ }^{18}$ are suggested to act in a similar manner, targeting the a/c-ring interface ${ }^{19}$.

As the a-subunit loop insertion is conserved within the Acinetobacter family but is absent or structurally diverse in other bacteria and the mitochondrial ATP synthase, the unique $a / c_{10}$ interface in A. baumannii represents a prime target for the development of highly specific inhibitors. BDQ belongs to a class of small molecules known as diarylquinolines (DARQs). A targeted screen of 700 DARQs yielded compounds that specifically inhibited the ATP synthase from S. aureus and Streptococcus pneumoniae but showed minimal activity towards M. tuberculosis ${ }^{20}$. These data demonstrate that inhibitors which bind at the c-ring or the $a / c_{10}$ interface can be optimised to target specific ATP synthases beyond Mycobacteria and future structure-based drug design may facilitate the development of compounds targeting this interface in ESKAPE pathogens ${ }^{1}$. The structure of the $A$. baumannii ATP synthase reveals novel and unique features of a bacterial ATP synthase and will inform future efforts to develop much needed antibiotics to treat MDR Acinetobacter. 


\section{Acknowledgments}

This work is dedicated to our late collaborator Éric Marsault (Sherbrooke University, Canada). We thank Paul Simpson and the Centre for Structural Biology (CSB) electron microscopy facility at Imperial College London for EM support. We are also grateful to Nora Cronin and the London Consortium for electron microscopy (LonCem) at the Crick Institute (London, UK), and the Electron Bio-Imaging Centre (eBIC) at Diamond Light Source (Harwell, UK) for their continued support and microscopy time. Suhail Islam is acknowledged for IT support. Nita Shah and Anaïs Menni are thanked for processing advice to JD. This work was funded by a Wellcome UK Investigator grant to TM [WT110068/Z/15/Z].

\section{Author contributions}

JKD, OLU and LPA grew cells, purified and optimised the protein sample. JKD collected and analysed data, built and analysed the structure and provided a manuscript draft. BPP analysed the structure, made figures, and wrote the final paper version. OLU performed biochemical assays. LPA and AF provided constructs, helped with Acinetobacter genetics, provided method sections and comments for the manuscript. MB built, refined and analysed the structure and contributed to the manuscript. TM obtained funding, analysed and interpreted data, wrote the paper and directed the project.

\section{References}

1. Mulani, M.S., Kamble, E.E., Kumkar, S.N., Tawre, M.S. \& Pardesi, K.R. Emerging Strategies to Combat ESKAPE Pathogens in the Era of Antimicrobial Resistance: A Review. Front Microbiol 10, 539 (2019). 
2. Duran-Manuel, E.M. et al. Clonal dispersion of Acinetobacter baumannii in an intensive care unit designed to patients COVID-19. J Infect Dev Ctries 15, 58-68 (2021).

3. Tacconelli, E. et al. Discovery, research, and development of new antibiotics: the WHO priority list of antibiotic-resistant bacteria and tuberculosis. Lancet Infect Dis $\mathbf{1 8}, \mathbf{3 1 8 -}$ 327 (2018).

4. Khoshnood, S. et al. Bedaquiline: Current status and future perspectives. J Glob Antimicrob Resist 25, 48-59 (2021).

5. Siroy, A. et al. Global comparison of the membrane subproteomes between a multidrug-resistant Acinetobacter baumannii strain and a reference strain. J Proteome Res 5, 3385-98 (2006).

6. Tiwari, V., Vashistt, J., Kapil, A. \& Moganty, R.R. Comparative proteomics of inner membrane fraction from carbapenem-resistant Acinetobacter baumannii with a reference strain. PLoS One 7, e39451 (2012).

7. Umland, T.C. et al. In vivo-validated essential genes identified in Acinetobacter baumannii by using human ascites overlap poorly with essential genes detected on laboratory media. mBio 3, e00113-12 (2012).

8. Wang, N., Ozer, E.A., Mandel, M.J. \& Hauser, A.R. Genome-wide identification of Acinetobacter baumannii genes necessary for persistence in the lung. mBio 5, e0116314 (2014).

9. Sobti, M. et al. Cryo-EM structures of the autoinhibited E. coli ATP synthase in three rotational states. Elife 5, e21598 (2016).

10. Guo, H., Suzuki, T. \& Rubinstein, J.L. Structure of a bacterial ATP synthase. elife 8, e43128 (2019). 
11. Sobti, M. et al. Cryo-EM reveals distinct conformations of E. coli ATP synthase on exposure to ATP. elife 8, e43864 (2019).

12. Hahn, A., Vonck, J., Mills, D.J., Meier, T. \& Kühlbrandt, W. Structure, mechanism, and regulation of the chloroplast ATP synthase. Science 360, eaat4318 (2018).

13. Abrahams, J.P., Leslie, A.G.W., Lutter, R. \& Walker, J.E. Structure at $2.8 \AA$ resolution of F1-ATPase from bovine heart mitochondria. Nature 370, 621-628 (1994).

14. Boyer, P.D. The ATP synthase-a splendid molecular machine. Annual Review of Biochemistry 66, 717-749 (1997).

15. Guo, H. et al. Structure of mycobacterial ATP synthase bound to the tuberculosis drug bedaquiline. Nature 589, 143-147 (2021).

16. von Ballmoos, C., Brunner, J. \& Dimroth, P. The ion channel of F-ATP synthase is the target of toxic organotin compounds. Proc Natl Acad Sci U S A 101, 11239-44 (2004).

17. Hong, S. \& Pedersen, P.L. ATP synthase and the actions of inhibitors utilized to study its roles in human health, disease, and other scientific areas. Microbiol Mol Biol Rev 72, 590-641 (2008).

18. Lamontagne Boulet, M. et al. Tomatidine is a lead antibiotic molecule that targets Staphylococcus aureus ATP synthase subunit c. Antimicrob Agents Chemother 62, e02197-17 (2018).

19. Martin-Galiano, A.J., Gorgojo, B., Kunin, C.M. \& de la Campa, A.G. Mefloquine and new related compounds target the $\mathrm{F}_{0}$ complex of the $\mathrm{F}_{0} \mathrm{~F}_{1} \mathrm{H}^{+}$-ATPase of Streptococcus pneumoniae. Antimicrob Agents Chemother 46, 1680-7 (2002).

20. Balemans, W. et al. Novel antibiotics targeting respiratory ATP synthesis in Grampositive pathogenic bacteria. Antimicrob Agents Chemother 56, 4131-9 (2012). 


\section{Figure legends}

Figure 1 - Structure of the $\mathrm{F}_{1} \mathrm{~F}_{\mathrm{o}}$-ATP synthase from $\boldsymbol{A}$. baumannii. A: Cryo-EM map of A. baumannii ATP synthase coloured by subunit as indicated (left). Surface representation of the corresponding atomic structure shown as spheres in orthogonal views in corresponding colours (right). The membrane is indicated by a light brown bar. B: Structure of the $F_{1}$ head including the central stalk; subunits $\alpha_{D P}, \beta_{D P}$ and $\alpha_{E}$ removed to reveal how subunits $\gamma$ (cyan) and $\varepsilon$ (pink) insert into the head. Close-up of the C-terminal extension of $\varepsilon$ inserting between $\beta_{T P}$ and $\alpha_{D P *}$ (not shown) showing the unwound turn of $\varepsilon{ }^{126} \mathrm{AQL}^{128}$ (yellow). C: Horizontal section of $F_{1}$ as indicated by dashed line in (B) including location of nucleotide binding sites. D: The catalytic nucleotide binding sites with EM map features corresponding to nucleotides and phosphates shown as mesh.

Figure 2 - Structure of $F_{0}$ motor and Acinetobacter specific features of the entry/exit channel sites. A: Ribbon representation of the a-subunit with the key conserved aR231 and the protonatable cD61 carboxylates in the $c_{10}$ ring shown in ball and stick representation. Periplasmic and cytoplasmic channels, are shown in maroon and purple, respectively. Arrow indicates direction of rotation during ATP synthesis. B: Surface rendering of the periplasmic channels in the a-subunits of $B$. taurus (dark blue) and A. baumannii (maroon) indicating alternate exit pathways. Equivalent residues forming the first mini-helix are coloured in purple. C: Comparison of $a / c_{10}$ interface in $A$. baumannii (yellow and light blue) and in $M$. smegmatis (dark grey and turquoise, PDB:7JGA) showing binding site of bedaquiline (BDQ) in M. smegmatis (light grey ball and sticks), proton carrying residues shown in dark red. Bottom panels are rotated by $180^{\circ}$ around the membrane normal D: Surface representation of c-ring (yellow) and a-subunit (translucent cyan) viewed from within the membrane plane. Insert - 
bioRxiv preprint doi: https://doi.org/10.1101/2021.07.10.451757; this version posted July 11, 2021. The copyright holder for this preprint

(which was not certified by peer review) is the author/funder. All rights reserved. No reuse allowed without permission.

structural alignment of a-subunits from A. baumannii, E. coli (PDB:6OQR) and M. smegmatis (PDB:7JGA) indicate extent of a-subunit loop insertion. K206 shown in stick representation. 
Figure 1
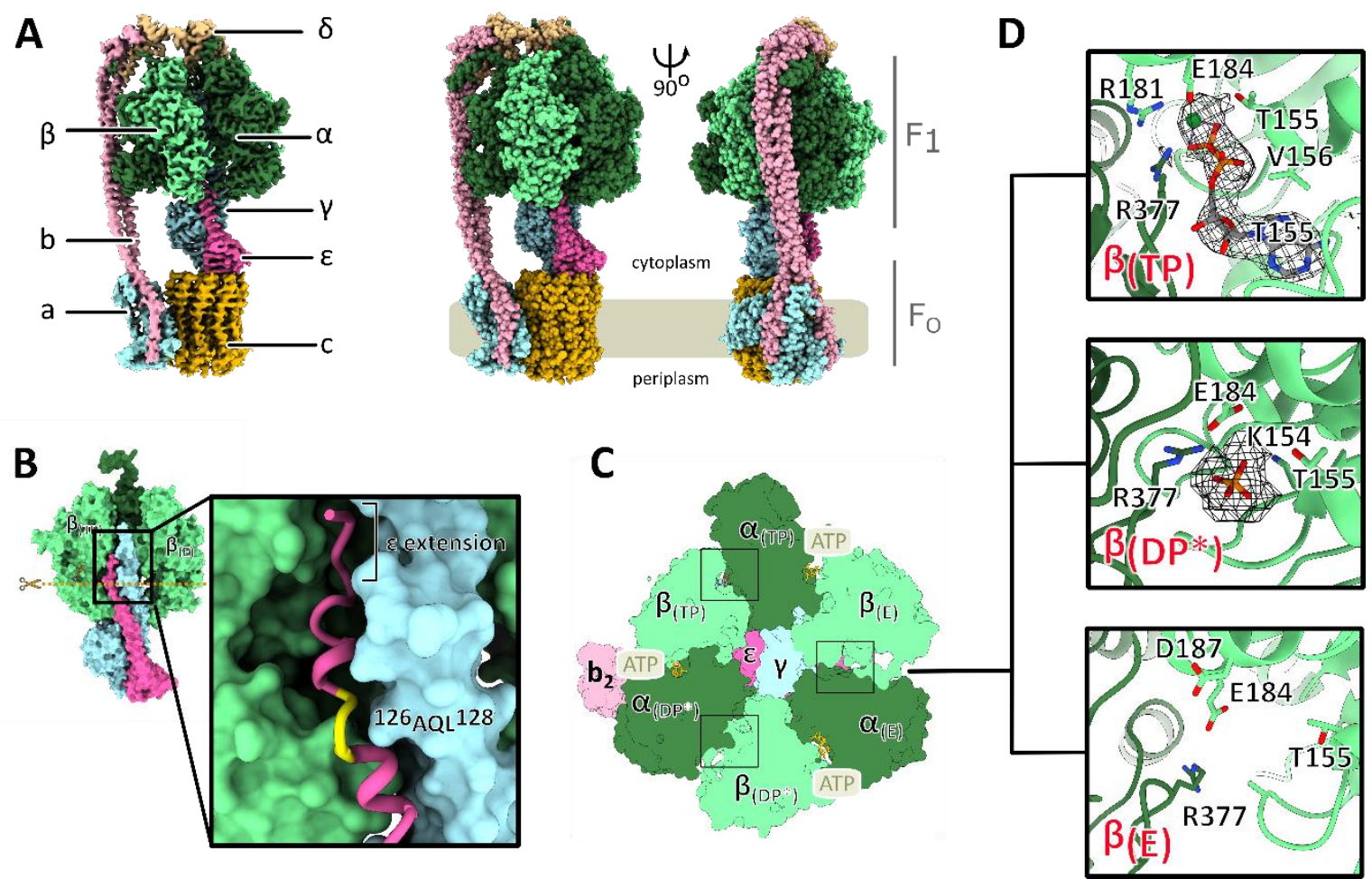


\section{Figure 2}

A

A

a subunit
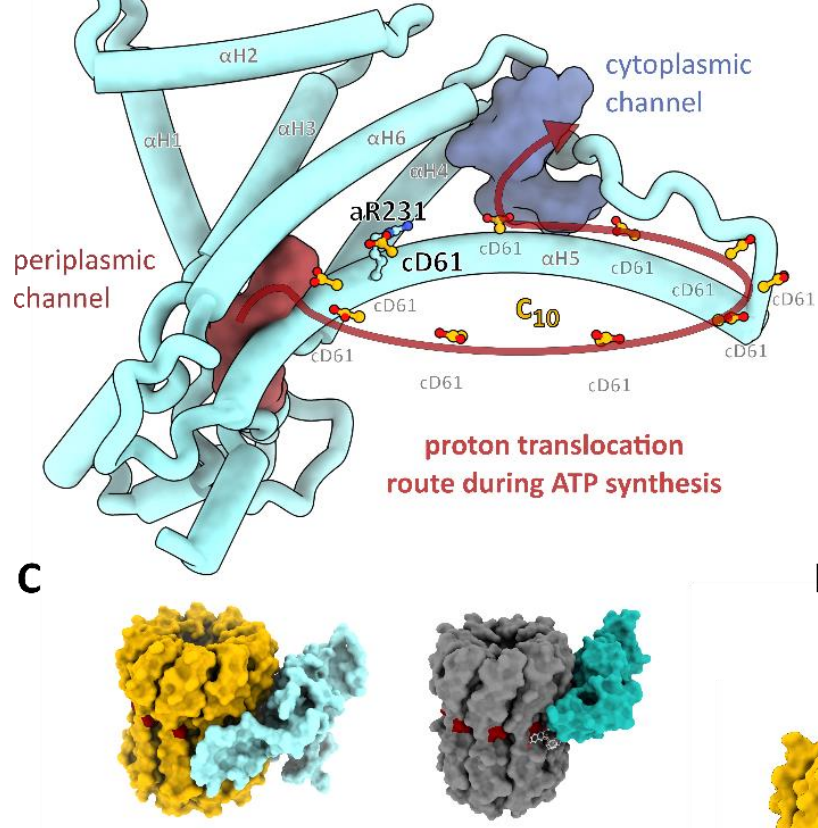

A. baumannii

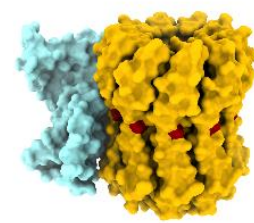

A. baumannii

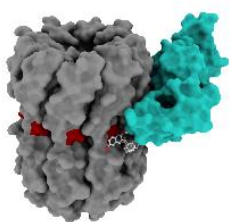

M. smegmatis $+B D Q$

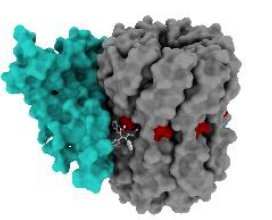

M. smegmatis $+B D Q$
B

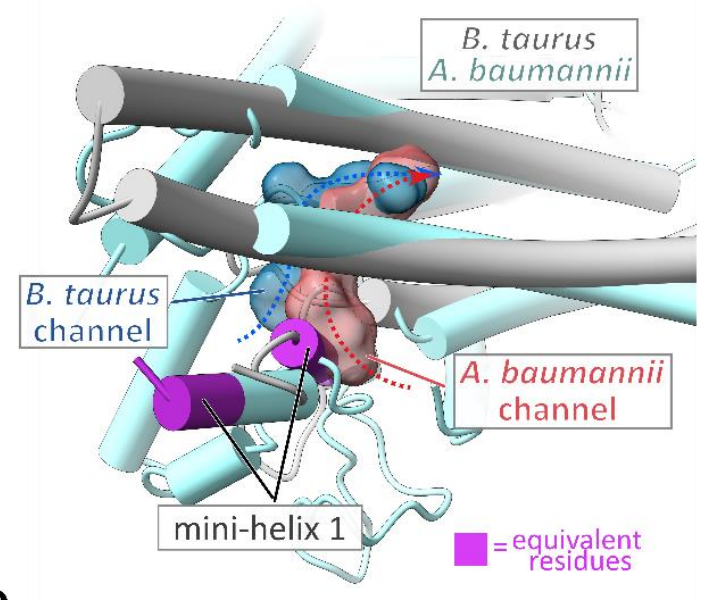

D

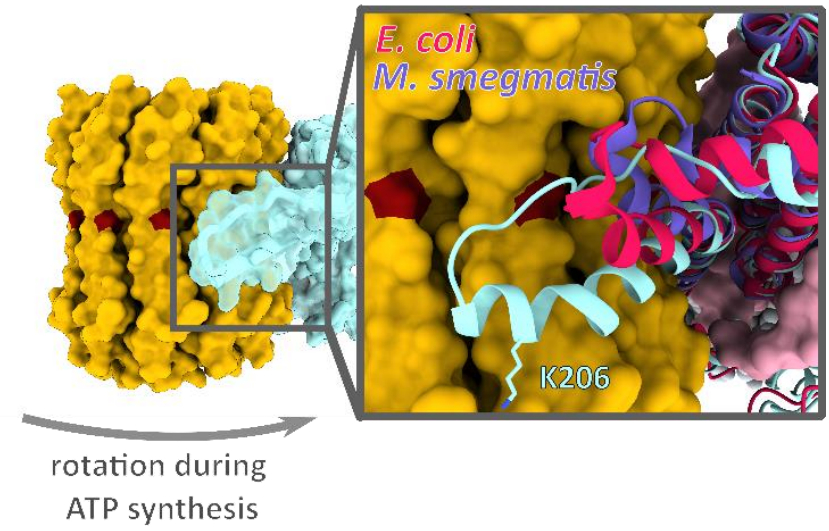

